\title{
PARADOXOS, O INFINITO E A INTUIÇÃO GEOMÉTRICA
}

\author{
Carlos G. González*

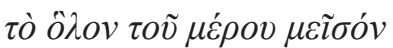 \\ Euclides, Elementos ${ }^{1}$
}

\section{RESUMO}

Analisam-se neste artigo alguns resultados matemáticos que já foram assinalados como contrários à intuição e se realiza uma pesquisa das possíveis causas desse caráter contraintuitivo. Nesse sentido, são estudados o Paradoxo de Galileu, a demonstração de Cantor de que o segmento tem a mesma quantidade de pontos que o quadrado e o Paradoxo de TarskiBanach. Primeiro é discutido o papel que o conceito de infinito e princípios como "o todo é maior que a parte" têm nesses paradoxos. Em segundo lugar, é estudada a influência que as intuições geométricas têm em alguns paradoxos e a relação delas com a concepção de geometria do Erlanger Programm de Felix Klein.

Palavras-chave: Paradoxos. Intuição. Erlanger Programm.

\begin{abstract}
This paper studies some mathematical results formerly characterized as opposed to the intuition and also carries out a query for the possible causes of this counter-intuitive feature. In this sense, both Tarski-Banach and Galileo's paradoxes are discussed and Cantor's proof that the segment and the square have the same number of points is analyzed. In particular, it is examined the role that the concept of infinity and such principles as "the whole is greater than the part" have in these paradoxes. Furthermore, the

\footnotetext{
* Doutor em Lógica e Filosofia da Ciência pela Universidade Estadual de Campinas. Professor Adjunto do Instituto de Filosofia da Universidade Federal de Uberlândia (UFU). E-mail: gonzalez@defil.ufu.br / gonzalcg@gmail.com

${ }^{1} \mathrm{O}$ todo [é] maior que a parte (HEATH; HEIBERG, 1908, p. 232).
} 
effect that geometrical intuitions have on some paradoxes as well as the relationship between these intuitions and the concept of geometry of the Erlanger Programm of Felix Klein are discussed.

Keywords: Paradox. Intuition. Erlanger Programm.

\section{Introdução}

Desde a antiguidade, certos resultados matemáticos foram considerados contrários à intuição. Por exemplo, os pitagóricos demonstraram que não existe uma medida comum, por menor que ela seja, entre a diagonal e o lado do quadrado, e Aristóteles escreveu sobre o espanto que causa tal resultado². No final do século XIX e o início do XX, apareceram na matemática vários resultados contraintuitivos e outros que geraram contradições, denominados genericamente de paradoxos.

Entretanto, a palavra paradoxo é utilizada de maneira ambígua na literatura ${ }^{3}$. Em particular, existem paradoxos que conduzem a contradições dentro de uma teoria (como o paradoxo de Russell no sistema de Frege ou o paradoxo de Burali-Forte nos primeiros sistemas de Cantor) ${ }^{4}$, outros que envolvem sérios problemas lógicos, linguísticos ou semânticos, como o paradoxo do mentiroso, e um terceiro tipo em que se encontram aqueles que não geram por si mesmos uma contradição, mas que são contrários à intuição, como o Paradoxo de Galileu ou o de Tarski-Banach. Este artigo ocupa-se com esse último tipo, apelando ao sentido etimológico de "paradoxo": que enfrenta a $\delta$ ó $\alpha$ [dóxa], que é contrário à aparência, que enfrenta a opinião.

Além disso, procuram-se as possíveis causas desse caráter contraintuitivo de certos paradoxos. Por um lado, é analisado o conceito de infinito, nas seções 2 e 3, e o papel que têm princípios sobre conjuntos finitos (como "o todo é maior que a parte") com relação ao conceito de

\footnotetext{
2 ARISTÓTELES, Metafisica, A2, 983a12-20.

${ }^{3}$ CLARK (2007) apresenta um panorama dos diversos paradoxos.

${ }_{4}$ Às vezes denominados de antinomias. Em Franenkel Bar-Hillel Levy (1973) é apresentada uma análise de alguns deles.
} 
infinito, nas seções 4 e 5, e, além disso, na seção 4 é discutido também o Paradoxo de Galileu. Um outro resultado considerado contraintuitivo, mas não referido na literatura como paradoxo, é analisado na seção 6: a demonstração de Cantor de que o segmento tem a mesma quantidade de pontos que o quadrado. O Paradoxo de Tarski-Banach é apresentado sinteticamente na seção 7 .

Por outro lado, estuda-se ainda a influência que intuições geométricas, relacionadas ao processo perceptivo, têm em alguns paradoxos, sendo as invariantes analisadas na seção 8 um exemplo típico disso. Na sequência, pesquisa-se a relação entre intuições geométricas e o conceito de geometria do Erlanger Programm de Felix Klein, nas seções 9 e 10.

\section{Experiência e finitude}

A experiência do dia a dia desenvolve-se com o uso de magnitudes finitas: medidas finitas, quantidades finitas, conjuntos com um número finito de elementos, etc. Além disso, diferentes operações aritméticas são aplicadas sobre essas magnitudes no cotidiano. Por exemplo, a soma é associada à operação material de juntar os elementos de dois conjuntos de objetos discretos, como também à medida resultante do comprimento total de duas varas colocadas em línea reta e a outras coisas. Quando existe um certo grau de abstração, além de associar propriedades, alguns princípios gerais são usados de maneira implícita ou explícita. Possivelmente "o todo é maior que a parte" seja um arquétipo desses princípios. Quando esse princípio é enunciado a uma pessoa que nunca havia pensado nele, nem meditado sobre as questões relativas à quantidade, é muito provável que o aceite como verdadeiro. O sentimento de uma certa evidência imediata, também denominado intuição $o^{5}$ geralmente acompanha essa afirmação. Será desconsiderado aqui o problema de até que ponto a experiência cotidiana contribui para essa intuição ${ }^{6}$, mas essa experiência atua de uma

\footnotetext{
5 A palavra intuição tem sido usada de diferentes maneiras em filosofia, mas o sentido de uma evidência imediata, de um sentimento subjetivo de certeza anterior a um raciocínio que o justifique tem sido comum em filosofia da matemática.

6 Esse problema constitui um dos pontos chaves do estudo da corrente da filosofia da matemática denominada naturalismo. Uma linha de estudos nesse sentido foi iniciada
} 
maneira confirmatória e consolida o sentimento de certeza sobre a verdade desses princípios. Analogamente, se é perguntado a uma pessoa sem nenhum aprofundamento matemático nem filosófico por que $2+2=4$, muito provavelmente oferecerá um exemplo concreto do dia a dia.

A generalização, cuidadosa ou imprudente, constitui um processo lógico habitual. Dessa maneira, muitas vezes princípios como "o todo é maior que a parte" são concebidos com a maior generalidade, como valendo universalmente. Há dois mil e trezentos anos atrás, um famoso matemático de nome Euclides escreveu um dos livros mais influentes e mais publicados da história do ocidente: um manual introdutório à geometria e aritmética elementar denominado Elementos (HEATH; HEIBERG, 1908). O seu caráter introdutório não faz dele um livro simples de $1 \mathrm{lr}^{7}$, pois o seu conteúdo principal são demonstrações matemáticas, como sequências encadeadas $^{8}$ de afirmações. Esse livro começa com definições e princípios aceitos sem demonstração. Ditos princípios estão divididos em dois

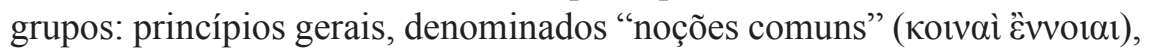

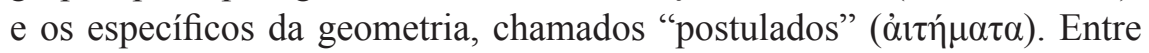
os postulados aparece: "por dois pontos passa uma única reta", e entre as noções comuns, podemos ler "o todo é maior que a parte" geral que é aplicado incondicionalmente para todo tipo de magnitudes: comprimentos de segmentos, medidas de ângulos, áreas, etc. Entretanto, foi essa generalidade atribuída ao princípio "o todo é maior que a parte" um dos motivos da rejeição das totalidades infinitas.

no século XIX por Helmholtz (CASSIRER, 1944). Willard Van Orman Quine é um dos principais representantes dessa corrente no século XX.

$7 \mathrm{Na}$ antiguidade contava-se a história, de verdade questionável, mas repetida através dos séculos, de que Ptolomeu perguntou a Euclides se não tinha uma maneira mais fácil de apreender geometria que os Elementos, ao qual Euclides respondeu: "não existe caminho real para a geometria".

$8 \mathrm{Ou}$, às vezes, pretendidamente encadeadas.

9 Nas palavras de Euclides: "desenhar uma linha reta de qualquer ponto para outro" (HEATH; HEIBERG, 1908, p. 195).

${ }^{10}$ Ibid., p. 232. 


\section{O conceito simples de infinito}

O que é infinito? A palavra parece dizer: "o que não tem fim”, "o que não termina", "o que não acaba", etc. "Infinito" foi usado em diferentes contextos, por exemplo, cedo na Grécia, o termo à $\pi \varepsilon$ ıov [ápeiron] foi usado num contexto metafísico por Anaximandro e, a partir dessa época, muitos outros filósofos utilizaram a palavra "infinito" em contextos metafísicos e teológicos.

Nos Elementos encontramos muitas vezes um procedimento simples, mas extremamente significativo da maneira grega de fazer geometria: prolongar um segmento de reta ${ }^{11}$. Sempre é possível prolongar um segmento sobre a reta na qual está incluído. Portanto, se essa prolongação sempre pode ser feita, ela pode ser feita ad infinitum, infinitamente. A situação é similar ao que acontece com a sequência dos números naturais $0,1,2,3, \ldots, n, \ldots$ sempre pode ser acrescentado um novo número, a sequência não tem fim, pode ser prolongada indefinidamente. Esse conceito é conhecido como infinito potencial ou infinito em potência ${ }^{12}$.

Um outro conceito de infinito é usado quando é concebida uma totalidade infinita, como no caso típico do conjunto dos números naturais. A noção geral de conjunto incluindo os conjuntos infinitos (e diferente de uma coleção finita de objetos concretos) é atribuída a Georg Cantor, na segunda metade do século XIX. Se pensarmos no conjunto dos números naturais, concebemos uma totalidade com infinitos elementos, um infinito atual ou infinito em ato ${ }^{13}$. Entretanto, a existência de "totalidades infinitas" foi debatida por séculos em diferentes contextos ${ }^{14}$, e muitas vezes rejeitada.

Quando nos referimos a conjuntos de uma maneira ingênua, podemos fazer a tentativa de contar os elementos de um conjunto $A$ usando

\footnotetext{
${ }^{11}$ Baseado no segundo postulado: "estender uma línea reta finita continuamente

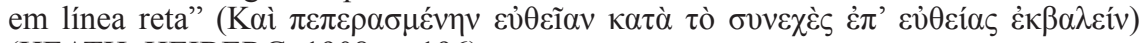
(HEATH; HEIBERG, 1908, p. 196).

12 Entretanto, os geômetras gregos preferiam não fazer menção do infinito nessas construções: Boyer menciona "Aristotle's statement that the mathematicians 'do not need the infinite or use it"” (BOYER; MERZBACH, 1991, p. 98).

${ }^{13}$ Cf. MOORE, 2001, p. 39-40.

${ }^{14} \mathrm{O}$ livrode Moore (2001) oferece um panorama dessas discussões.
} 
os números naturais. Se conseguirmos contar os elementos de $A$, então $A$ é finito. Caso contrário, $A$ é infinito, pois não terminamos nunca de contar os seus elementos.

Na moderna teoria de conjuntos, na teoria axiomática de conjuntos de Zermelo-Fraenkel, Z F, a existência de conjuntos infinitos é estabelecida por meio de um axioma, o Axioma de Infinito, que determina a existência do conjunto dos números naturais. Além disso, em ZF cada número natural $n$ é também um conjunto, mais precisamente, $n$ é um conjunto de $n$ elementos: 0 é o conjunto vazio, 1 é um conjunto de um elemento, 2 é um conjunto de dois elementos, etc. A partir disso, podemos contar os elementos de um conjunto $A$, ou seja, podemos colocar os números naturais maiores que zero em pares com os elementos de $A$ :
O primeiro elemento de $A a_{1}$
O segundo elemento de $A a_{2}$
O terceiro elemento de $A a_{3}$

Se esse processo termina com um número natural $n$, então o conjunto é finito, tem $n$ elementos e pode ser estabelecida uma correspondência um a um com $n$ entendido como conjunto de $n$ elementos. Se esse processo não tem fim e não podemos estabelecer essa correspondência com um número natural, então o conjunto é infinito. Essa é a definição habitual de conjunto infinito.

\section{4 "O todo é maior que a parte" e o Paradoxo de Galileu}

Ao longo dos séculos, o princípio "o todo é maior que a parte" foi aceito e aplicado em diferentes contextos. Em particular, num argumento denominado Paradoxo de Galileu ${ }^{15}$, ele foi usado para negar a existência de totalidades infinitas.

Já nos Elementos de Euclides, o princípio, “o todo é maior que a parte", é usado com extrema generalidade e aplicado em diferentes

\footnotetext{
${ }^{15}$ Aparece em Duas novas ciências (GALILEI, 1811), mas está fora do interesse deste artigo se foi ou não enunciado originalmente por Galileu.
} 
contextos. Quando essa obra é comparada com os Fundamentos de Geometria de Hilbert (HILBERT, 1913), faz-se notório ter sido aumentado consideravelmente o grupo de postulados métricos ${ }^{16}$, enquanto não aparece mais o axioma do todo e a parte. Na realidade, a obra de Euclides utiliza muitas vezes, sem mencionar, características métricas do espaço euclidiano, que foram as explicitadas por Hilbert ${ }^{17}$. No contexto euclidiano, o axioma "o todo é maior que a parte", trabalha como um coringa de diferentes propriedades métricas do espaço. Igualmente, através da história, esse axioma foi interpretado com a maior generalidade.

O Paradoxo de Galileu faz um uso particular desse princípio ${ }^{18}$, sob uma forma que poderíamos explicitar como "a quantidade de elementos de uma parte de um conjunto é menor que a quantidade do todo". O argumento conclui que "maior", "menor" e "igual" não podem ser aplicados ao infinito ${ }^{19}$. Como consequência disso, pode ser negada a existência do infinito atual em matemática. Hoje poderia ser pensado como a negação da existência de conjuntos infinitos. Uma reformulação das linhas principais desse paradoxo poderia ser a seguinte. Para chegar a uma contradição, suponha que exista o conjunto dos números inteiros positivos:

16 Fundamentalmente o grupo quatro dos postulados de congruência.

${ }^{17}$ Os Elementos também contêm o postulado: "todos os ângulos retos são iguais", que faz referência a medidas. Mas, por exemplo, não contêm nenhum postulado sobre características métricas de segmentos. Curiosamente, essa falta de postulados métricos é usada implicitamente até a atualidade. Por exemplo, o Postulado 2 dos Elementos (ver nota 11) é enunciado como: "Para todo segmento $\mathrm{AB}$ e para todo segmento $\mathrm{CD}$, existe um único ponto $\mathrm{E}$ tal que $\mathrm{B}$ está entre $\mathrm{A}$ e $\mathrm{E}$, e o segmento $\mathrm{CD}$ é congruente com o segmento BE" "For every segment $\mathrm{AB}$ and for every segment $\mathrm{CD}$ there exists a unique point $\mathrm{E}$ such that $\mathrm{B}$ is between A and E and segment CD is congruent to segment BE", (GREENBERG, 1994, p. 15). Para ver que não são equivalentes, consideremos um segmento $[\mathrm{A}, \mathrm{B}]$ sobre o intervalo aberto $(\mathrm{M}, \mathrm{N}):[\mathrm{A}, \mathrm{B}] \subseteq(\mathrm{M}, \mathrm{N})$. O intervalo $[\mathrm{A}, \mathrm{B}]$ sempre pode ser estendido segundo o postulado original de Euclides, mas a versão moderna citada é falsa nesse modelo.

${ }^{18} \mathrm{O}$ argumento começa com Simplício confundido pelo fato de que uma parte de um segmento parece ter mais pontos que o segmento inteiro, enquanto ambos têm uma infinidade de pontos: "bisogna confessare trovarsi nel medesimo genere una cosa maggior dell'infinito, perché la infinità de i punti della linea maggiore eccederà l'infinità de i punti della minore" (GALILEI, 1811, p. 59).

${ }^{19}$ Galileu afirma: "ed in ultima conclusione, gli attributi di eguale maggiore e minore non aver luogo ne gl'infiniti, ma solo nelle quantità terminate”, (GALILEI, 1811, p. 61-62). 


$$
P=\{1,2,3,4, \ldots, n, \ldots\}
$$

Então também deveria existir o conjunto dos quadrados desses números:

$$
Q=\left\{1,4,9,16, \ldots, n^{2}, \ldots\right\}
$$

Entretanto, podemos estabelecer uma correspondência um a um (uma função bijetora) entre $x$ e $x^{2}$ :

$$
\begin{gathered}
f(1)=1 \\
f(2)=4 \\
f(3)=9 \\
\vdots \\
f(n)=n^{2}
\end{gathered}
$$

Se interpretamos a palavra "igual" como existindo uma correspondência um a um, então temos que uma parte, o conjunto $Q$ dos quadrados, é igual ao todo, o conjunto $P$, de modo que estaria em contradição com o axioma mencionado.

\section{Cantor e o salto entre o finito e o infinito}

O processo de abstração utiliza muitas vezes o mecanismo de separar propriedades, focalizando algumas e desconsiderando outras. Por exemplo, temos uma maçã vermelha, como objeto sensível do qual temos experiência. Separamos a propriedade da cor das outras propriedades da maçã: ser uma fruta, ter açúcar, ter uma massa e um peso, etc. e consideramos somente a cor, tomando em conta unicamente "o vermelho" ou "vermelhalidade" da maçã. Uma vez separada, focalizada, essa propriedade pode ser usada para classificar objetos segundo a cor, de modo que poderíamos agrupar os objetos da mesma cor, ficando a nossa maçã no grupo dos objetos de cor vermelha. Entretanto, poderíamos 
ter focalizado numa outra propriedade: "ser uma fruta", "ser um objeto aproximadamente esférico", "pesar menos de um quilograma", "conter açúcar", etc., resultando uma outra classificação de objetos.

No início da teoria de conjuntos, Cantor fez menção à abstração para suas noções de ordinal e de cardinal. Dado um conjunto $A$, ele diz que o número ordinal de $A$, que escreve $A$, resulta de considerar (focalizar) o tipo de ordem e desconsiderar todas as outras características dos objetos de $A^{20}$. Segundo Cantor, ainda pode ser aplicada uma nova abstração, na qual seria desconsiderado o tipo de ordem, ficando somente a quantidade de elementos, o número cardinal de $A$, que escreve $A$, indicando que foi realizado um duplo processo de abstração ${ }^{21}$. Do ponto de vista formal, Cantor definiu que dois conjuntos têm o mesmo número cardinal; se pode ser estabelecida uma correspondência um a um entre os elementos de ambos e associou sua definição com a ideia intuitiva de quantidade de elementos, resultando no enunciado:

1. Dois conjuntos nos quais pode ser realizada uma correspondência um a um entre os seus elementos têm a mesma quantidade de elementos.

O paradoxo de Galileu baseia-se na contradição existente entre o enunciado 1 e "o todo é maior que a parte", reinterpretado como:

2. A quantidade de elementos de uma parte de um conjunto é menor que a quantidade de elementos do todo.

Intuitivamente, parece que ambos os princípios valem para conjuntos finitos. Entretanto, como Cantor queria formular uma noção de quantidade para os conjuntos infinitos, sendo consciente de duas coisas:

- o enunciado 2 é incompatível com os conjuntos infinitos e

\footnotetext{
${ }^{20}$ CANTOR, 1932, p. 297.

${ }^{21}$ CANTOR, 1932, p. 282.
} 
- um processo de abstração deve selecionar, focalizar, algumas propriedade e desconsiderar outras.

Cantor decidiu focalizar o enunciado 1 e desconsiderar o 2. Numa atitude de extrema inovação e coragem abandonou o princípio euclidiano que reinou por mais de dois mil anos.

Refletindo sobre esse fato, vemos que o Paradoxo de Galileu nasce de supor que uma propriedade aplicada ingenuamente aos conjuntos finitos pode ser extrapolada aos conjuntos infinitos ${ }^{22}$. Por outro lado, Cantor não está disposto a generalizar para os conjuntos infinitos todas as propriedades dos conjuntos finitos, pois estabelece que o enunciado 2 não vale para os conjuntos infinitos. Dessa maneira, a passagem das propriedades dos conjuntos finitos para as dos conjuntos infinitos não é realizada por uma simples generalização, mas por um salto, uma descontinuidade, que estabelece que nem toda propriedade dos conjuntos finitos vale também para os conjuntos infinitos. Há um processo de abstração que desconsidera uma propriedade dos conjuntos finitos quando se quer abranger também os conjuntos infinitos. Em outras palavras, para poder desenvolver a matemática dos conjuntos infinitos, Cantor realiza intencionalmente um processo de abstração, estabelecendo que existe, no sentido conceitual, um salto entre o finito e o infinito, motivo pelo qual não podemos simplesmente generalizar para os conjuntos infinitos as propriedades dos conjuntos finitos ${ }^{23}$.

Quando não é considerado esse salto entre o finito e o infinito, ou seja, quando as nossas intuições sobre magnitudes finitas são ingenuamente extrapoladas para todos os conjuntos, incluindo os infinitos, então se obtêm contradições e resultados contraintuitivos. Por exemplo, podemos ficar perplexos diante do fato de que uma parte de um conjunto infinito tenha

\footnotetext{
${ }^{22}$ Galileu nega explicitamente que propriedades atribuídas ao finito possam ser diretamente extrapoladas ao infinito: "Queste son di quelle difficoltà che derivano dal discorrer che noi facciamo col nostro intelletto finito in torno a gl'infiniti, dandogli quelli attributi che noi diamo alle cose finite e terminate", (GALILEI, 1811, p. 59).

${ }^{23}$ Cf. HILBERT, 1926, p. 166-167 e 172.
} 
a mesma quantidade de elementos que o conjunto total. Ou podemos usar princípios que somente são aplicáveis a conjuntos finitos e que conduzem a contradições quando usados com conjuntos infinitos.

No sentido em que este trabalho utiliza o termo "paradoxo", alguns paradoxos surgem de desconsiderar ingenuamente esse salto entre o finito e o infinito, tal como acontece no Paradoxo de Galileu. Em outras palavras, resulta contraintuitivo porque enunciados que valem somente para conjuntos finitos são extrapolados para os infinitos.

Entretanto, nem todos os resultados matemáticos envolvendo conjuntos infinitos resultam contraintuitivos, paradoxais somente por esse salto entre o finito e o infinito. Como veremos, as intuições geométricas têm um papel fundamental em outros paradoxos, sendo exemplo típico o Paradoxo de Tarski-Banach, e em outros resultados contraintuitivos, como a prova de Cantor da correspondência entre os pontos do segmento e do quadrado.

\section{O segmento e o quadrado}

Uma vez aceitos os conjuntos infinitos e as correspondências que podem ser estabelecidas, para cada conjunto infinito $A$, entre $A$ e uma parte própria de $A$, fica a pergunta: até onde seremos levados por esse tipo de procedimento? Haverá consequências contraintuitivas? Um exemplo histórico pode ser útil para esclarecer esses problemas.

Suponha-se um sistema de coordenadas do plano e o segmento

$$
s=[0 ; 1]=\{x \in \mathbf{R}: 0 \leq x \leq 1\}
$$

desenhado sobre o eixo dos $x$. Pode-se associar o primeiro ponto de $s$ com 0 , o último com 1 , e todos os intermediários com desenvolvimentos decimais $0, \mathrm{~d}_{1} \mathrm{~d}_{2} \mathrm{~d}_{3}, \ldots$ Por outro lado, considerando o quadrado,

$$
\mathrm{Q}=\{<\mathrm{x}, \mathrm{y}>: x \in \mathbf{R}, y \in \mathbf{R}, 0 \leq x \leq 1 \text { e } 0 \leq y \leq 1\},
$$

cada ponto pode ser descrito como um par de números. Para estabelecer uma correspondência entre os pontos do quadrado e os do segmento, deveria ser atribuído a cada número real $x$ um par de números 
reais $\langle y, z>$, tais que $0 \leq y \leq 1$ e $0 \leq z \leq 1$ e vice-versa. A ideia fundamental do procedimento de Cantor $^{24}$ pode ser exemplificada com um número:

$$
p_{s}=0,2536849405
$$

correspondendo ao par :

$$
\begin{aligned}
& p_{x}=0,23890 \ldots \\
& p_{y}=0,56445 \ldots
\end{aligned}
$$

ou seja, os dígitos do ponto do segmento $p_{s}$ usam alternativamente os dígitos das duas coordenadas do ponto do quadrado $p_{x}$ e $p_{y}$ : o primeiro dígito de $p_{s}$ depois da vírgula (ou seja, 2) corresponde ao primeiro de $p_{x}, \mathrm{o}$ segundo de $p_{s}$ ao primeiro de $p_{y}(5)$, o terceiro de $p_{s}$ ao segundo de $p_{x}(3)$, o quarto de $p_{s}(6)$, ao segundo de $p_{y}$, etc.

Esse resultado já foi indicado como "contraintuitivo" 25 . Provavelmente, a intuição geométrica indicaria que o quadrado deva ter mais pontos que o segmento, pois o segmento é uma parte do quadrado e, segundo o axioma euclidiano, o todo é maior que a parte. Entretanto, um outro tipo de intuições parecem ser contraditas por esse resultado: o segmento tem dimensão 1, enquanto o quadrado tem dimensão 2: como é possível que um objeto geométrico de uma dimensão possa ser transformado num objeto de duas dimensões? Na realidade, a demonstração de Cantor vai além, pois os pontos do segmento podem também ser rearranjados para formar um cubo e objetos geométricos de $n$ dimensões para qualquer inteiro positivo $n$. De alguma maneira, essa demonstração questiona alguma noção intuitiva referida ao número de dimensões. Se considerarmos que a noção de dimensão é essencial à geometria, então essa prova seria antigeométrica, porquanto oposta a certas intuições geométricas.

\footnotetext{
${ }^{24}$ Esse procedimento é explicado numa carta a Dedekind, que critica a prova. Cantor (1932) desenvolve uma prova mais complexa do mesmo resultado. Posteriormente, Hausdorff apresentou uma prova simplificada. Ver Dauben (1990) para os detalhes.

${ }^{25}$ Cf. DAUBEN, 1990, p. 25.
} 


\section{A decomposição paradoxal de uma esfera}

Outro famoso resultado considerado contraintuitivo é uma demonstração do particionamento de uma esfera conhecido como Paradoxo de Tarski-Banach. De maneira sintética ${ }^{26}$, uma esfera é dividida em um número finito de partes. Depois, rearranjando essas partes (sem as esticar nem comprimir), são definidas duas esferas de raio igual à anterior.

Seria realmente contraditório se dividíssemos a esfera da maneira que é cortada uma laranja com uma faca ${ }^{27}$ e juntássemos os pedaços para formar duas laranjas de tamanho igual à original. A contradição surgiria porque os pedaços cortados com a faca teriam definido um volume, de modo que a soma dos volumes dos pedaços tem que dar sempre o mesmo resultado e, portanto, o enunciado "o todo é maior que a parte" geraria uma contradição (sendo o todo correspondente ao volume das duas laranjas e a parte, ao volume de uma delas). Entretanto, se os pontos forem manipulados usando procedimentos conjuntistas, como na demonstração de Cantor da seção 6, não existe contradição, já que o procedimento não preserva volumes. Na realidade, na demonstração de Tarski-Banach são definidos conjuntos de pontos para os quais não pode ser definido um volume $^{28}$. Cortar uma laranja com uma faca é visto intuitivamente como um procedimento geométrico que preserva volumes. A noção geométrica de volume também contribui para o caráter contraintuitivo, paradoxal, desse resultado: a construção utilizada no Paradoxo de Tarski-Banach também pode ser não geométrica em algum sentido que deverá ser especificado.

\section{Geometria e invariantes}

Nossa percepção de objetos tem como passo inicial e fundamental o reconhecimento de que diferentes percepções correspondem ao mesmo

\footnotetext{
${ }^{26}$ Ver Jech, 1973 para os detalhes.

${ }^{27}$ Pode ser idealizado um procedimento geométrico análogo a cortar uma laranja com uma faca, usando planos e semiplanos para particionar uma esfera.

${ }^{28}$ Essas afirmações sobre volumes poderiam ser melhores especificadas, usando teoria da medida (HALMOS, 1970), pois, como assinala JECH (1973), o ponto chave da demonstração consiste em definir conjuntos não mensuráveis, conjuntos para os quais não pode ser definida uma medida.
} 
objeto $^{29}$. No caso mais simples, da percepção de uma figura geométrica, se perguntarmos a uma pessoa qual é a forma de um determinado azulejo e indicamos ostensivamente qual é o objeto ao qual nos estamos referindo, e supondo que esse objeto tem forma aproximadamente quadrada, seguramente o nosso interlocutor afirmará que a forma é quadrada. Entretanto, provavelmente a imagem na retina dessa pessoa não seja um quadrado, devido fundamentalmente à perspectiva. Mudando o ângulo e a distância do objeto percebido, essa pessoa reafirmará que é um quadrado. Talvez, um pouco importunado expressará: “se eu já falei que é um quadrado". Embora, a mudança no ângulo e na distância tenha produzido percepções muito diferentes, ambas são identificadas com o mesmo objeto e com a mesma forma geométrica. Podemos afirmar, portanto, que quando passarmos de uma para outra percepção, ambas referem-se ao mesmo objeto, ou seja, o objeto permanece invariante. Do ponto de vista geométrico acontece algo similar com relação à forma. A forma quadrada (por mais que não seja quadrada na retina) permanece invariante. Em tal sentido, Cassirer afirma:

Conceitos matemáticos são independentes de qualquer limite que possa ser imposto pela percepção. $\mathrm{O}$ conceito geométrico abraça a totalidade e variedade ilimitada de modificações que suporta uma figura espacial quando é sujeita a transformações determinadas ${ }^{30}$.

Tais invariantes geométricos acontecem toda hora na vida cotidiana. Quando o pedreiro fez o piso, ele tirou o azulejo da caixa e supôs, corretamente, que a forma e o tamanho dele não iriam mudar com o transporte até o seu lugar definitivo no chão. Do ponto de vista geométrico a forma e o tamanho do azulejo são invariantes para a translação e a rotação que são necessárias para colocar o azulejo na sua posição definitiva. $\mathrm{Na}$ geometria euclidiana, que reinou absoluta durante dois mil anos, a

\footnotetext{
${ }^{29}$ Isso gera o problema da "perceptual constancy", analisado em Cassirier (1944, p. 9s).

30 "Mathematical concepts are independent of any limits that might be imposed upon perception. The geometrical concept embraces and comprehends the totality and unlimited variety of modifications which a spatial figure undergoes when it is subjected to certain transformations" (CASSIRER, 1944, p. 17).
} 
forma e a área de uma figura são invariantes por translações e rotações: transladando e rotacionando uma figura no plano euclidiano, o resultado será sempre uma figura da mesma forma e da mesma área. Se um triângulo tem um ângulo reto, poderemos transladá-lo a qualquer parte do plano e rotá-lo de qualquer maneira que continuará tendo um ângulo reto. $\mathrm{Na}$ geometria euclidiana, também a simetria também é uma transformação que respeita invariantes. Suponha que rotacionamos um triângulo isósceles pelo seu eixo de simetria: obtemos um triângulo com os mesmos valores para ângulos, lados, áreas, etc. Ou seja, a área e a medida dos ângulos permanecerão invariantes por simetrias.

A geometria euclidiana tem mais transformações que respeitam invariantes. Suponha a seguinte construção que transforma um triângulo em outro com o dobro do comprimento dos lados. Estendamos dois lados adjacentes para o dobro de seu comprimento. Agora, desenhemos um segmento que una os extremos dos dois lados estendidos. Temos um novo triângulo, mas a medida dos ângulos permaneceu invariante, enquanto áreas e comprimentos não. Então poderíamos definir as transformações para as quais as medidas dos ângulos das figuras são consideradas uma invariante. Claramente, a translação, a rotação e a simetria estão entre essas transformações. Entretanto, algumas outras transformações, como a construção do triângulo indicada acima, também seriam transformações que têm como invariante a medida dos ângulos.

Nas geometrias não euclidianas de Riemann e Lobachevski, a construção do triângulo indicada acima não tem como invariante os ângulos das figuras. Por exemplo, na geometria de Riemann, a medida dos ângulos aumenta em tal construção. Para ver isso, consideremos um análogo da geometria de Riemann: a geometria esférica, que em lugar de ser exemplificada no plano, utiliza a superfície de uma esfera. Um triângulo infinitamente pequeno ${ }^{31}$ teria a soma dos ângulos interiores igual a dois retos, como na geometria euclidiana. Mas qualquer triângulo desenhado na esfera teria a soma dos seus ângulos maior que dois retos. Desenhemos um triângulo a partir do Polo Norte, com um ângulo reto. Estendamos os dois lados até o Equador e desenhemos o terceiro lado do triângulo sobre

${ }^{31}$ Por exemplo, entendido como um limite. 
o Equador. Teríamos um triângulo de três ângulos retos. Entretanto, os ângulos das figuras seriam invariantes para translações, rotações e simetrias, como acontece com a geometria euclidiana. Por exemplo, os ângulos de um triângulo não mudam pelo fato de o triângulo ser transladado de uma parte para outra da esfera.

Para a geometria projetiva, os ângulos não são mais invariantes por translações. Essa geometria está inspirada na noção de perspectiva. Pensemos um quadrado visto de frente, cuja imagem na retina é também quadrada. Agora mudemos o ângulo de visão e a imagem do quadrado será um trapézio, mudando a magnitude dos ângulos. Em outras palavras, a translação mudou a medida dos ângulos, diferentemente do que acontece com as geometrias euclidianas de Riemann e de Lobachevski. Ou seja, a medida dos ângulos não é uma invariante para a translação na geometria projetiva. Entretanto, a translação da geometria projetiva nunca poderá transformar um quadrado num pentágono, nem numa esfera, porque a quantidade de ângulos de uma figura é uma invariante para a translação nessa geometria.

Tanto na geometria de Riemann como na de Lobachevski, a curvatura do espaço é constante. Motivados muitas vezes pela noção de espaço da teoria geral da relatividade, foram estudados espaços geométricos cuja curvatura não é constante. Num espaço cuja curvatura não é constante, a medida dos ângulos de um triângulo não é invariante para translações. No lugar de uma esfera, que tem curvatura constante, pensemos um balão alongado, mais curvo numas partes que em outras: transladar um triângulo de um lugar para outro poderia mudar os seus ângulos.

$\mathrm{Na}$ topologia podem ser definidos espaços topológicos nos quais um homomorfismo pode transformar um quadrado num círculo. Para esses homomorfismos, a quantidade de ângulos não seria mais uma invariante. Entretanto, a topologia estuda um outro tipo de invariantes: os homomorfismos que transformam curvas sem buracos (ou seja, sem descontinuidades) em outras curvas sem buracos, sendo a noção de continuidade uma invariante para esses homomorfismos. 


\section{Procedimentos geométricos e o Erlanger Programm}

O Paradoxo de Tarski-Banach nos coloca perante a pergunta: certas técnicas de teoria de conjuntos, em particular as que usam poderosos procedimentos para manipular conjuntos de pontos, podem ser admitidas ou não em geometria? Na geometria grega e, em particular, na geometria de Euclides, os procedimentos aceitos fundamentavam-se no uso de regra e compasso. Mas esses instrumentos somente podiam ser usados em procedimentos que gerassem transformações com as invariantes da geometria euclidiana. Ou seja, a geometria euclidiana está caracterizada pelas transformações que respeitam as invariantes da dita geometria. Generalizando essa ideia, podemos nos perguntar o que é que caracteriza uma geometria. Nas palavras de Poincaré:

O que é, afinal, uma geometria? É o estudo de um grupo de operações formado pelos deslocamentos que podem ser feitos a uma figura sem a deformar ${ }^{32}$.

O desenvolvimento das geometrias não euclidianas, da geometria projetiva e da topologia, entre outros avanços, gerou uma visão mais geral e abstrata do que pode ser uma geometria. Aparece o conceito de variedade (manifold) e é usada a teoria de grupos para definir um grupo de transformações, estando Felix Klein e Sophus Lie à cabeça dessas pesquisas ${ }^{33}$.

A grande contribuição de Klein e Lie à geometria está baseada em que, dada uma determinada geometria, as transformações que respeitam um conjunto dado de invariantes têm a estrutura matemática denominada "grupo", constituindo um grupo de transformações. Por exemplo, para qualquer transformação do grupo que transforme o objeto $A$ no objeto $B$, existe uma transformação do grupo que converte $B$ em $A$. Ademais, existe uma transformação identidade no grupo, que transforma qualquer objeto $A$ no mesmo objeto $A$, as transformações são associativas, etc.

32 “Qu'est-ce en effet qu'une Géométrie? C'est l'étude du groupe d'opérations formé par les déplacements que l'on peut faire subir à une figure sans la déformer" (POINCARÉ apud GRAY, 1992, p. 40).

${ }^{33}$ Cf. ROWE, 1992. 
Segundo expressa Klein (1872), no seu famoso Erlanger Programm, devem ser estudadas aquelas propriedades das figuras que permanecem invariantes para todas as transformações de um grupo dado:

Dados uma variedade e um grupo de transformações sobre ela, devem ser pesquisadas aquelas propriedades das figuras pertencentes a essa variedade que não mudam pelas transformações do grupo ${ }^{34}$.

Dessa maneira, uma geometria define um grupo de transformações que respeita certas invariantes.

Por exemplo, na geometria euclidiana podemos aplicar transformações como a translação e a rotação. Mas quando transladamos ou rotacionamos uma figura, temos que certas quantidades, como sua área, o comprimento de seus lados, os seus ângulos, etc. permanecem constantes, ou seja, invariantes. Uma outra geometria está caracterizada por um outro grupo de transformações e um outro conjunto de invariantes.

\section{Geometria, invariantes e intuição}

Segundo vimos, um primeiro tipo de paradoxos está constituído por uma extrapolação do finito para o infinito, como com o Paradoxo de Galileu. Nesta seção analisaremos o papel das intuições geométricas em outros paradoxos, como o de Tarski-Banach.

Qual é o fundamento para dizer que algo não é geométrico? Uma resposta possível é a do Erlanger Programm de Klein: uma geometria está caracterizada por um grupo de transformações e um conjunto de invariantes para esse grupo de transformações. As construções e demonstrações que utilizam procedimentos que não respeitam as invariantes são não geométricas nesse sentido.

O procedimento cantoriano da seção 6 é não geométrico no sentido que a dimensão não é uma invariante (não é preservada) pela transformação

\footnotetext{
34 "Es ist eine Mannigfaltigkeit und in derselben eine Transformationsgruppe gegeben; man soll die der Mannigfaltigkeit angehörigen Gebilde hinsichtlich solcher Eigenschaften untersuchen, die durch die Transformationen der Gruppe nicht geändert werden.” (KLEIN, 1921, p. 463). Cf. BIRKHOFF; BENNETT, 1988, p. 150.
} 
do segmento num quadrado, como acontece nas transformações aceitas em várias geometrias. Também é não geométrica num sentido mais profundo, pois Cantor demonstrou que não existe transformação do segmento no quadrado que preserve continuidade, um dos conceitos fundamentais da topologia. O paradoxo de Tarski-Banach fundamenta-se na não preservação de volumes, que é uma invariante fundamental da geometria euclidiana. Num sentido mais técnico, conjuntos não mensuráveis não são geométricos, pois a medida é uma das invariantes fundamentais de uma geometria.

Poderíamos dizer que por volta de 1870 acontece uma divisão do trabalho. Por um lado, Cantor renuncia a princípios como "o todo é maior que a parte" e às intuições geométricas para desenvolver uma teoria de conjuntos abstrata, cujos conjuntos de pontos vão muito além da geometria. Por outro lado, Klein e Lie começam a estudar os grupos de transformações que caracterizam as geometrias. Durante dois milênios as intuições geométricas foram usadas implicitamente, com uma certa ingenuidade, mas no século XIX várias intuições geométricas foram substituídas por princípios explícitos: o axioma de Pasch, os axiomas de congruência de Hilbert e a noção de continuidade são alguns exemplos disso. Com relação a esse último, Dedekind afirma em Continuidade e Números Irracionais:

até as mais rigorosas apresentações do cálculo diferencial fundamentam suas demonstrações não na continuidade, mas, em vez dela, elas recorrem, com mais ou menos consciência, a representações geométricas, ou sugeridas pela geometria ${ }^{35}$.

Ou seja, a definição e o pressuposto da continuidade eram omitidos porque eram usados implicitamente na base de intuições geométricas. Em outras palavras, o uso implícito de uma intuição geométrica é substituído pela definição de continuidade e enunciados que usam esse conceito. Esse fato tem muito a ver com a posição de Cantor e Dedekind, que pretende

\footnotetext{
35 “auch die strengsten Darstellungen der Differentialrechnung gründen ihre Beweise nicht auf die Stetigkeit, sondern sie appellieren entweder mit mehr oder weniger Bewußtsein an geometrische, oder durch die Geometrie veranlaßte Vorstellungen" (DEDEKIND, 1932, p. 316).
} 
eliminar as intuições geométricas e, quando necessário, substituí-las por definições explícitas. Nesse sentido, Cassirer (1944) afirma:

Se for considerado esse desenvolvimento, deve, com efeito, ser admitido que a intuição perdeu sua posição lógica predominante e que caiu sua importância como um meio de demonstração geométrica ${ }^{36}$.

Mas de maneira alguma a esse desenvolvimento histórico deve ser atribuída a eliminação as intuições geométricas nem a sua insignificância. Nas palavras de Klein:

É da intuição que nós derivamos os dados que, com uma idealização apropriada, são submetidos a tratamento lógico ${ }^{37}$.

\section{Como também:}

Desenvolver uma consideração geométrica de um modo puramente lógico, sem que a figura a que ela se refere permaneça frente a meus olhos é, em todo caso, impossível para $\mathrm{mim}^{38}$.

Em síntese: a intuição persiste, mas o sentido contraintuitivo de alguns paradoxos pode ser esclarecido pela análise de que certos procedimentos podem ser considerados não geométricos no sentido técnico no qual Klein definiu o que é uma geometria.

\footnotetext{
36 "If one considers this development, one must indeed admit that intuition has lost its predominant logical position and that it has sunk in importance as a means of geometrical demonstration" (CASSIRER, 1944, p. 14).

37 "It is from intuition that we derive the data which, in appropriate idealization, are subject to logical treatment." Ibid.

38 "Eine geometrische Betrachtung rein logisch zu führen, ohne mir die Figur, auf welche dieselbe Bezug nimmt, fortgesetzt vor Augen zu halten, ist jedenfalls mir unmöglich.”(DEDEKIND, 1932, p. 381). Cf. CASSIRER, 1944 , p. 14.
} 


\section{Conclusões}

A tese principal do artigo é que existem (pelo menos) dois tipos de ideias intuitivas que tornam paradoxais algumas afirmações. O primeiro tipo está constituído por não respeitar o salto existente entre o finito e o infinito. O exemplo típico disso é o Paradoxo de Galileu. O segundo tipo, como Tarski-Banach, resulta paradoxal por se opor a determinadas intuições geométricas: seria uma construção não geométrica.

Quando uma área é definida como um conjunto de pontos e são aplicadas técnicas poderosas de teoria de conjuntos para a manipulação desses conjuntos de pontos, então podem ser criados procedimentos para os quais não existam as invariantes geométricas habituais, como áreas, ângulos ou comprimentos. Inclusive, pode ser estabelecida a existência de conjuntos, como os conjuntos não mensuráveis, cujo comportamento resulta contrário às intuições geométricas, por não respeitar as invariantes de uma geometria.

Em outras palavras, se uma transformação usando técnicas conjuntistas é aplicada a conjuntos de entes geométricos, pode acontecer que não tenha as invariantes que requer uma determinada geometria, como a geometria euclidiana que requer que a translação tenha áreas, comprimentos, ângulos, etc. como invariantes. No sentido do Erlanger Programm, essas técnicas não seriam geométricas, coincidindo com o sentido contraintuitivo que possuem algumas delas. Por exemplo, o sentido contraintuitivo do paradoxo de Tarski-Banach surgiria não porque esse resultado é contrário às intuições sobre operações sobre conjuntos de pontos, senão porque se opõe às intuições geométricas envolvidas.

$\mathrm{O}$ procedimento idealizado por Cantor, que estabelece uma correspondência um a um entre um segmento e um quadrado, foi considerado contraintuitivo, fundamentalmente, porque o conceito de dimensão não é invariante para ele. Muito mais grave que modificar áreas, comprimentos ou formas, o procedimento cantoriano modifica o número de dimensões, pois o segmento tem dimensão 1, enquanto o quadrado tem dimensão 2. Essa transformação enfrenta radicalmente as intuições geométricas.

$\mathrm{Na}$ geometria euclidiana e nos desenvolvimentos iniciais da análise matemática, muitas intuições geométricas causaram o uso implícito 
e inconsciente de afirmações matemáticas. Por um lado, matemáticos como Pasch, Hilbert, Dedekind e outros explicitaram os enunciados implícitos em intuições geométricas. Por outro lado, Klein, Lie e outros introduziram uma visão mais geral e mais abstrata do que é uma geometria e os procedimentos geométricos. Dessa maneira, intuições não geométricas correspondem muitas vezes a procedimentos não geométricos.

\section{Referências}

ARISTÓTELES. Aristotle's Metaphysics. A Revised Text with Introduction and Commentary by W. D. Ross, Oxford: Clarendon Press, 1975.

BIRKHOFF, G.; BENNETT, M. K. Felix Klein and his "Erlanger Programm". In: ASPRAY, W.; KITCHER, P. (Ed.). History and philosophy of modern mathematics. Minneapolis: The University of Minnesota Press, 1988. p. 145-176.

BOI, L.; FLAMENT, D.; SALANSKIS, J.-M. (Ed.). 1830-1930: A Century of Geometry. Epistemology, History and Mathematics. Berlin: Springer, 1992. (Lecture Notes in Physics, 402).

BOYER, C. B.; MERZBACH, U C. A History Of Mathematics. 2 ed. New York: John Wiley \& Son, 1991.

CANTOR, G. Gesammelte Abhandlungen mathematischen und philosophischen Inhalts. Berlin: Springer, 1932.

CASSIRER, E. The concept of group and the theory of perception. Philosophy and Phenomenological Research, Buffalo, N. Y., v.5, n.1, p. 1-36, Sept. 1944.

CLARK, M. Paradoxes from A to Z. 2. ed. London: Routledge, 2007.

DAUBEN, J. W. Georg Cantor: His Mathematics and Philosophy of the Infinite. Princeton: Princeton University Press, 1990.

DEDEKIND, R. Gesammelte mathematische Werke. Braunschweig: F. Vieweg \& Sohn, 1932.

FRAENKEL, A.; BAR-HILLELL, Y.; LEVY, A. Foundations Of Set Theory. Amsterdam: Elsevier, 1973. 
GALILEI, G. Discorsi e dimostrazioni matematiche intorno a due nuove scienze attenenti alla mecanica, \& i movimenti locali. In: . Milano: Società tipografica de'classici italiani, 1811. (Opere di Galileo Galilei, v. 8). GRAY, J. Poincare and Klein - groups and geometries. In: BOI, L.; FLAMENT, D.; SALANSKIS, J.-M. (Ed.). 1830 - 1930: A Century of Geometry. Epistemology, History and Mathematics. Berlin: Springer, 1992. p. 35-44. (Lecture Notes in Physics, 402).

GREENBERG, M. J. Euclidean and Non-Euclidean Geometries. Development and History. 3. ed. New York: W. H. Freeman, 1994.

HALMOS, P. R. Measure Theory. Berlin: Springer, 1970.

HEATH, S.; HEIBERG, J. The thirteen books of Euclid's Elements. Translated with Introduction e Commentary by Sir Thomas L. Heath from the Greek Text of J. Heiberg. Cambridge: Cambridge University Press, 1908. HILBERT, D. Grundlagen der Geometrie. 4. ed. Leipzig: B. G. Teubner, 1913.

. Ueber das Unendliche. Mathematische Annalen, Leipzig, v. 95, n. 1, p. 161-190. 1926.

JECH, T. The axiom of Choice. Amsterdam: Elsevier, 1973.

KLEIN, F. Vergleichende Betrachtungen uber neuere geometrische Forschungen. Programm zum Eintritt in die philosophische Facultat und den Senat der k. Friedrich-Alexanders - Universitat zu Erlangen. Erlangen: Deichert, 1872.

. Gesammelte mathematische Abhandlungen. Ersten Band. Berlin: J. Springer, 1921.

MOORE, A. W. The infinite. London: Routledge, 2001.

ROWE, D. E. Klein, Lie, and the "Erlanger Programm". In: BOI, L.; FLAMENT, D.; SALANSKIS, J.-M. (Ed.). 1830 - 1930: A Century of Geometry. Epistemology, History and Mathematics. Berlin: Springer, 1992. p. 45-54. (Lecture Notes in Physics, 402). 
